\title{
ON THE MEASURE OF WELL-DISTRIBUTED SEQUENCES
}

\section{ALAN ZAME}

0 . Introduction. A sequence $\left(x_{n}\right)$ of numbers is said to be uniformly distributed modulo 1 [henceforth we will just write u.d.] if for every subinterval $I$ of the unit interval the following relation holds:

$$
\left.\lim _{N \rightarrow \infty} \frac{1}{N} \text { (\# of } x_{n} \text { in } I(\operatorname{modulo} 1), \text { with } 1 \leqq n \leqq N\right)=\|I\|
$$

where $\|I\|$ is the length of $I$. It is immediate that if $\left(^{*}\right)$ holds then so does

$$
\text { (**) } \left.\lim _{N \rightarrow \infty} \frac{1}{N}\left(\# \text { of } x_{n} \text { in } I \text { (modulo } 1\right), k \leqq n \leqq N+k-1\right)=\|I\|
$$

for each positive integer $k$. If $\left(^{* *}\right)$ holds uniformly in $k$ for each subinterval $I$ of the unit interval, then $\left(x_{n}\right)$ is said to be well distributed modulo 1 (w.d.).

Recently there have been several articles (see [3], [5], [6], [11][14]) concerned with the following question: suppose $\left(r_{n}\right)$ is a fixed increasing sequence of numbers. What can be said about the measure of the set $W$ of $x$ such that the sequence $\left(r_{n} x\right)$ is w.d.? If inf $\left(r_{n}-r_{n-1}\right)$ $>0$, it follows by a result of Koksma [10] that the sequences $\left(r_{n} x\right)$ are u.d. for almost all $x$ (in the sense of Lebesgue measure). The sequences $(n x)$ are w.d. for all irrational $x$; on the other hand, if $a$ is an integer then it is easy to see that $\left(a^{n} x\right)$ can never be w.d. This last observation has led a number of authors to investigate lacunary sequences $\left(r_{n}\right)$, i.e., sequences of positive numbers with the property that

$$
\liminf _{n \rightarrow \infty}\left(r_{n} / r_{n-1}\right)>1 \text {. }
$$

In particular, under some additional conditions on the $\left(r_{n}\right)$ it has been shown that $\mu(W)=0$ (where $\mu$ is Lebesgue measure). This is the case, for instance, if the ratios $r_{n} / r_{n-1}$ are integral, or if the $r_{n}$ are integers and the ratio $r_{n} / r_{n-1}$ tends to infinity. It is also true if $r_{n}=\alpha^{n}, \alpha$ a real number. These results have led to the conjecture that for any lacunary sequence $\left(r_{n}\right)$ we have $\mu(W)=0$. It is the purpose of this paper to prove this conjecture and to discuss some other examples.

Presented to the Society, November 12, 1966; received by the editors June 12, 1966 and, in revised form, December 21, 1966. 
We will also note that these results are valid in certain topological groups.

\section{The main result.}

THEOREM 1. Let $\left(r_{n}\right)$ be a lacunary sequence and let $W$ be the set of $x$ for which the sequence $\left(r_{n} x\right)$ is w.d. Then $\mu(w)=0$.

Proof. We will first prove the following lemma from which the theorem will follow easily.

LEMma 2. Suppose that, for $n \geqq 2, r_{n} / r_{n-1} \geqq r>2$ and that $I$ is any closed subinterval of the unit interval for which $r\|I\| \geqq 2$. Then, given any integer $k$, for almost all $x$ there exists an integer $n(=n(x))$ such that each of the terms $r_{n+1} x, r_{n+2} x, \cdots, r_{n+k} x$ lies in I (modulo 1 ).

Proof. Let $\theta$ be any bounded interval and let $S_{k}$ be the above set. We will show that $\mu\left(\mathcal{O} \cap S_{k}\right) \geqq \mu(I)^{k}(1 / 3)^{k} \mu(\mathcal{O})$ which in turn implies, by a well known result, that $\mu\left(-S_{k}\right)=0$. First of all, notice that there exists $r_{m}$ such that $\left\|r_{m} \Theta\right\| \geqq 2$. Hence there are disjoint subintervals $I_{1}, \cdots, I_{p}$ of $\mathcal{O}$ such that

(i) $r_{m} I_{j} \equiv I(\bmod 1)$,

(ii) $\left(\mu \bigcup_{j} I_{j}\right) \geqq(1 / 3) \mu(I)(\mu \Theta)$, (where if $U=[a, b]$ is an interval, then $\alpha U=[\alpha a, \alpha b])$. Since $\mu(I) r \geqq 2$ it follows that $\left\|r_{m+1} I_{j}\right\| \geqq 2$, so each $I_{j}$ contains disjoint subintervals $I_{j}^{1}, \cdots, I_{j}^{p}(p=p(j))$ such that

(i) $r_{m+1} I_{j}^{t} \equiv I(\bmod 1)$,

(ii) $\mu\left(\bigcup_{t} I_{j}^{t}\right) \geqq(1 / 3) \mu\left(I_{j}\right) \mu(I)$, so that

$$
\mu\left(\bigcup_{t, j} I_{j}^{t}\right) \geqq(1 / 3)^{2} \mu(I)^{2} \mu(\mathcal{O}) .
$$

Continuing this process by induction our assertion follows.

PROOF OF THE THEOREM. Without loss of generality we may assume that $\inf _{n \geqq 2}\left(r_{n} / r_{n-1}\right) \geqq r>1$. There exists an integer $N$ such that $(1 / 2)$ $(1 / N) r^{N}>2$. By the lemma, for almost all $x$ and any integer $k$ there exists $n=n(x)$ such that

$$
r_{N(n+1)} x, r_{N(n+2)} x, \cdots, r_{N(n+k)} x
$$

each lie in the interval $[0,(1 / 2 N)]=\mathcal{u}(\operatorname{modulo} 1)$. However, if $\left(r_{n} x\right)$ is w.d. there exists $n_{0}$ : the fraction of the number of terms from $r_{p} x, \cdots, r_{p+m} x$ which lie in $u$ differs from $1 / 2 N$ by at most $1 /(4 N)$ for all $m \geqq n_{0}$ and all $p \geqq 1$. However, if $x$ is as above, then for $k \geqq n_{0}$ and $n=n(x)$ at least $k$ of the $N k-N+1$ terms $r_{N n+N} x, \cdots, r_{N n+N k} x$ lie in $\mathcal{u}$, a fraction $>1 / N$ and $1 / N-1 /(2 N)=1 /(2 N)>1 /(4 N)$, so $\left(r_{n} x\right)$ is not well distributed and the theorem follows. 
2. Some further results. If $p(x)$ is any nonconstant polynomial, then the sequence $(p(n) x)$ is w.d. except for at most a countably infinite number of $x$. (For a proof of this result, see [9].) This contrasts with the case $\left(a^{n} x\right)$ ( $a$ integral) which, as mentioned before, is never w.d. and raises the question as to further information in both the lacunary case and nonlacunary case. In the lacunary case we have the following

TheOREM 3. Suppose $\lim \left(r_{n} / r_{n-1}\right)=r>1$ (where $r$ may be infinite). Then (i) if $r=\infty$, the Hausdorff dimension of $W$ is 1.

(ii) if $r$ is finite, W may be empty or (uncountably) infinite.

Proof. (i) Let $\left(x_{n}\right)$ be any w.d. sequence. Using the same method as Erdös and Taylor [16] we may show that the set of $x$ for which $r_{n} x \rightarrow x_{n}$ (modulo 1) has dimension 1 , and it is known that if $\left(x_{n}\right)$ is w.d. and $y_{n} \rightarrow x_{n}$ (modulo 1) then $\left(y_{n}\right)$ is w.d. so the result follows.

(ii) An example satisfying the first part has already been given. For the second part, let $\left(n_{k}\right)$ be any "rapidly increasing" sequence of integers (say, $\left(n_{k}\right)$ lacunary) and let $\mathcal{O}=\sum_{k}(1 / 2)^{n_{k}}$. Then it is easy to see, since $\theta$ is irrational and the nonzero terms in the binary expansion of $\theta$ are "rare," that the sequence $\left(\left(2^{n}+n\right) \theta\right)$ is w.d.

Notice that the set of $\theta$ given in part (ii) has Hausdorff dimension 0 . It would be interesting to know whether a set of positive dimension (dimension 1) can be constructed.

In the other direction we have the following

THEOREM 4. There exists an increasing sequence of positive integers $\left(n_{k}\right)$, with $1 \leqq n_{k+1}-n_{k} \leqq M$ (for some constant $\left.M\right)$, such that the sequence $\left(n_{k} x\right)$ is not w.d. for any $x$.

Proof. Consider the interval $I=[1 / 5,1 / 4]$. Notice that if none of the terms $n x,(n+1) x, \cdots,(n+4) x$ lies in the interval $u=[1 / 8,3 / 8]$ $(\bmod 1)($ for $x \in I)$, then $(n+5) x$ lies in $I(\bmod 1)$. We now construct a sequence of positive integers $\left(n_{k}\right)$ as follows:

Choose $n_{1}, n_{2}, 1 \leqq n_{2}-n_{1} \leqq 6$, such that $n_{i}(1 / 5) \in \mathcal{u}(\bmod 1), i=1,2$. Notice that we may take $n_{2} \leqq 12$ and that if $|x-1 / 5|<1 /(8 \cdot 12)$ then $n_{i} x \in \mathcal{O}=[0,1 / 2](\bmod 1)$, i.e., if

$$
1 / 5 \leqq x \leqq 1 / 5+1 /(8 \cdot 12) \text {. }
$$

Next, find $n_{3}, n_{4}: 1 \leqq n_{3}-n_{2} \leqq 6,1 \leqq n_{4}-n_{3} \leqq 6$, such that $n_{i} v \in \mathcal{U}$ $(i=3,4)$, where $v=1 / 5+1 /(8 \cdot 12)$. Then, $n_{4} \leqq 4 \cdot 6$ and if $|y-v|$ $\leqq 1 /(8 \cdot 24)$ then $n_{i} y \in \mathcal{O}(\bmod 1), i=3,4$. Continue this process. At the $k$ th step we obtain integers $n_{2 k-1}, n_{2 k}$ such that

(i) $1 \leqq n_{2 k-1}-n_{2 k-2} \leqq 6$,

(ii) $1 \leqq n_{2 k}-n_{2 k-1} \leqq 6$, 
(iii) $n_{i} z \in \mathcal{U}, i=2 k-1,2 k$, where $z=1 / 5+(1 /(8 \cdot 12)) S$, where $S=1$ $+\frac{1}{2}+\cdots+1 /(k-1)$. Hence, if

$$
1 / 5+S \leqq x \leqq 1 / 5+S+(1 / 8 \cdot 12)(1 / k)
$$

then $n_{j} x \in \mathcal{O}(\bmod 1), j=2 k-1,2 k$. Since $\sum 1 / n$ diverges we may cover the interval $I$ in this manner, using only finitely many integers $n_{i}$, say $i=1, \cdots, m$.

We next find integers $n_{m+1}, n_{m+2}, n_{m+3}$, with gaps as before, such that $n_{i}(1 / 5) \in \mathcal{U}, i=m+1, m+2, m+3$. Again, $n_{m+3} \leqq 6(m+3)$ and if $|x-1 / 5| \leqq 1 /(8 \cdot 6(m+3))$ then $n_{i} x \in \mathcal{O}(\bmod 1), i=m+1, m+2$, $m+3$. We repeat this process, eventually covering the interval $I$. At that next stage, we repeat the construction, this time for four consecutive terms lying in $\mathfrak{u}(\bmod 1)$. We finally obtain a sequence $\left(n_{k}\right)$, $1 \leqq n_{k}-n_{k-1} \leqq 6$, with the property that if $x \in I$ and if $n$ is any positive integer, then there exists an integer $p$ such that $n_{i} x \in(\bmod 1)$ for $i=p, p+1, \cdots, p+m$. It is clear that if $x \in I$, then $\left(n_{i} x\right)$ is not w.d. Let $z$ be any real number. If $z$ is rational then $\left(n_{i} z\right)$ is not u.d. If $z$ is irrational, then some in tegral multiple of $z$, say $s z$, lies in $I \bmod 1$, so that the sequence $\left(n_{i} s z\right)$ is not w.d. But, from Weyl's criterion for w.d. sequences it then follows that $\left(n_{i} z\right)$ is not w.d. and the theorem is proved.

3. Distribution in compact abelian groups. Let $G$ be any compact abelian group, $\nu$ normalized Haar measure. We can define the notions of a u.d. and a w.d. sequence as before, with open sets replacing intervals and Haar measure replacing Lebesgue measure; equivalently we may require

$$
\lim \sum f\left(x_{k}\right)=\int f d \nu
$$

for every $f \in C(G)$, or even only for every continuous character $\chi$. Suppose now that $G$ is second countable (so that the character group $X$ is countable) and that $G$ is connected (so that the character group $X$ is torsion-free (see [8, Theorem 24.25])) and let $\chi$ be any character, $\chi \neq 1, T$ the unit circle. Then $\chi(G)=T, \chi^{-1}$ is measurable and, if $U$ is any measurable subset of $T$ then $\mu(U)=\nu\left(\chi^{-1}(U)\right)$ since $\nu \chi^{-1}$ is a translation-invariant measure on $T$. We can therefore deduce some distribution theorems on $G$ from the corresponding theorems on $T$. For example, if $\left(n_{k}\right)$ is a strictly increasing sequence of positive integers, then for $\nu$-a.a. $x$ in $G$ the sequence of $\left(x^{n_{k}}\right)$ is u.d. If $\left(n_{k}\right)$ is a lacunary sequence of integers, then for $\nu$-a.a. $x$ the sequence $\left(x^{n_{k}}\right)$ is not w.d. The first-mentioned result generalizes a result of Halmos and 
Samelson [7] and Eckmann [4] and could also be proved directly by imitating Koksma's proof [10] using the fact that $X$ is torsion-free.

\section{BiBLIOGRAPHY}

1. P. C. Baayen and G. Helmberg, On families of equi-u.d. sequences in compact spaces, Math. Ann. 161 (1965), 255-278.

2. J. G. van der Corput, Zur Gleichverteilung mod Eins, Acta Math. 56 (1931), 373-456.

3. A. F. Dowidar and G. M. Petersen, The distribution of sequences and summability, Canad. J. Math. 15 (1963), 1-10.

4. B. Eckmann, Über monothetische Gruppen, Comment. Math. Helv. 16 (1943 /1944), 249-263.

5. P. Gerl, Konstruktion gleichverteilung Punktfolgen, Monatsh. Math. 69 (1965).

6. - Eine Bemerkung zur gleichmassigen Gleichverteilung mod 1, Monatsh. Math. 70 (1966), 106-110.

7. P. R. Halmos and H. Samelson, On monothetic groups, Proc. Nat. Acad. Sci. U.S.A. 28 (1942), 254-258.

8. E. Hewitt and K. A. Ross, Abstract harmonic analysis. I, Springer, Berlin, 1963.

9. B. Lawton, A note on well distributed sequences, Proc. Amer. Math. Soc. 10 (1959), 891-893.

10. J. F. Koksma, Ein mengentheoretischer Satz über die Gleichverteilung mod Eins, Compositio Math. 2 (1935), 250-258.

11. B. H. Murdoch, A note on well-distributed sequences, Canad. J. Math. 17 (1965), 808-810.

12. G. M. Petersen and M. T. McGregor, On the structure of well distributed sequences, Nieuw Arch. Wisk. 11 (1963), 64-67.

13. - On the structure of well-distributed sequences. II, Indag. Math. 26 (1964), 477-487.

14. - On the structure of well-distributed sequences. III, Indag. Math. 28 (1966), 42-49.

15. H. Weyl, Über die Gleichverteilung von Zahlen mod Eins, Math. Ann. 77 (1916), 313-352.

16. P. Erdös and S. J. Taylor, On the set of points of convergence of a lacunary trigonometric series and the equidistribution properties of related sequences, Proc. London Math. Soc. 7 (1957), 598-615.

UNIVERSITY OF MIAMI 